\title{
Nonlocal damage mechanics
}

\author{
Milan Jirásek \\ Department of Mechanics \\ Faculty of Civil Engineering \\ Czech Technical University in Prague \\ 16629 Prague, Czech Republic \\ Milan.Jirasek@epfl.ch
}

\begin{abstract}
This paper starts with an overview of models that can provide an objective description of highly localized inelastic deformation. Basic ideas behind the integral and differential formulations of nonlocal models are explained using a simple isotropic damage model as a typical example. Regularizing effect of nonlocal enhancements is documented by one-dimensional localization analysis. The paper then focuses in detail on integral-type nonlocal damage models. The main issues addressed here include the choice of the internal variable to be averaged, the influence of boundaries, and various computational aspects ranging from efficient implementation of nonlocal averaging to adaptive techniques.

RÉSUMÉ. Cet article commence par un résumé des modèles avec une description objective des déformations inélastiques fortement localisées. Les principes de bases des modèles non locaux, intégral et gradient, sont illustrés dans le cas simple du modèle d'endommagement isotrope. Les effets de la régularisation des modèles enrichis sont illustrés sur la localisation dans le cas unidimensionnel. Dans la suite, nous présentons en détail l'approche intégrale des modèles non locaux. Un intérêt particulier est apporté sur le choix de la variable interne à régulariser, sur l'influence de la prise en compte des conditions aux limites et sur divers aspects du calcul numérique comme l'implantation efficace des modèles régularisés ou les techniques adaptatives.
\end{abstract}

KEYWORDS: strain localization, softening, localization limiters, nonlocal damage, failure simulations.

MOTS-CLÉS : localisation de la déformation, adoucissement, limiteurs de la localisation, endommagement non local, calcul à la rupture.

REGC - 11/2007. Damage and fracture in geomaterials, pages 993 à 1021 


\section{Classification of models for localized inelastic deformation}

In many structures subjected to extreme loading conditions, the initially smooth distribution of strain changes into a highly localized one. Typically, the strain increments are concentrated in narrow bands while the major part of the structure experiences unloading. The size of the localized band and its evolution depend on details of the material microstructure, in particular on the size and spacing of major heterogeneities and on the specific failure mechanism that develops for a given type of loading. Propagation and coalescence of microdefects in the localization band can eventually lead to the formation of a displacement discontinuity, e.g., of a macroscopic stress-free crack or a sharp slip line.

Mathematical and numerical models of concrete failure must correctly reflect the energy dissipated in the fracture process zone. As shown in (Jirásek, 2007), this is not the case if the stress-strain laws with softening is used within the standard continuum theory. Numerical results obtained with such models suffer from pathological sensitivity to the spatial discretization, e.g. to the size of finite elements. Upon mesh refinement, the energy dissipated by the numerical model decreases and tends to extremely low values, sometimes even to zero. As a remedy, one of the following approaches can be used:

1) The cohesive crack model admits the presence of a strong discontinuity (jump in the displacement field) and describes softening by a traction-separation law, which relates the traction transmitted by the crack to the crack opening.

2) The crack band model represents the process zone (and later a macroscopic crack) by a band of highly localized strain, separated from the surrounding material by two weak discontinuities (surfaces across which the strain field has a jump). Since the width of the numerically resolved band is controlled by the size of finite elements, the softening part of the stress-strain law must be adjusted according to the element size.

3) Regularized models are based on generalized continuum theories that incorporate a characteristic length and prevent localization of strain into an arbitrarily small volume. Since the enrichments enforce a certain minimum width of the numerically resolved process zone, they are called localization limiters. Examples of such generalized continua include nonlocal integral or differential (gradient-enriched) formulations.

Here we focus on regularized models, and in particular on nonlocal models of the integral type, because gradient models are covered by another paper in this journal issue (Simone, 2007).

From the mathematical point of view, the basic classes of models mentioned above are characterized by different levels of regularity (smoothness) of the underlying kinematic description; see Figure 1.

Cohesive crack models incorporate strong discontinuities, i.e., jumps in displacements across a discontinuity curve (in two dimensions) or discontinuity surface (in 
(a)
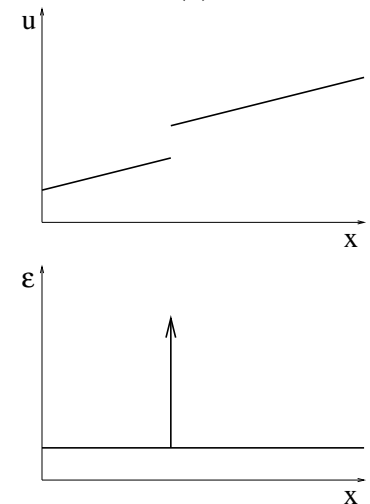

(b)
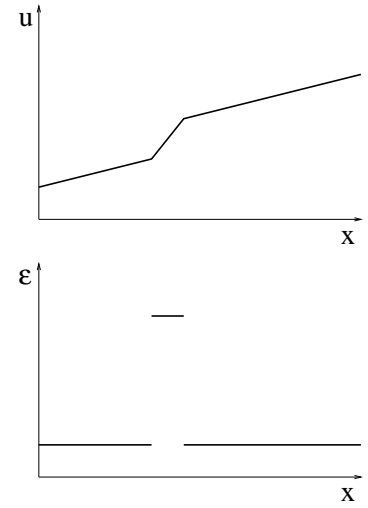

(c)
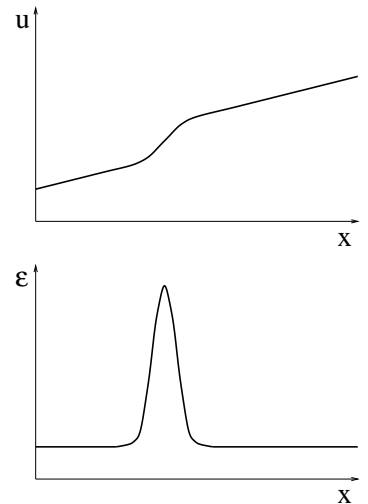

Figure 1. Kinematic description with (a) strong discontinuity, (b) weak discontinuities, (c) no discontinuities

three dimensions). The strain field, $\varepsilon(\boldsymbol{x})$, then consists of a regular part, obtained by standard differentiation of the displacement field, and a singular part, having the character of a multiple of the Dirac delta distribution. This is schematically shown for the one-dimensional case in Figure 1a. In physical terms, the strong discontinuity corresponds to a sharp crack (not necessarily a stress-free one).

Another possible kinematic description, exploited by the crack band models, represents the region of localized deformation by a band of a small but finite thickness, separated from the remaining part of the body by two weak discontinuities, i.e., curves or surfaces across which certain strain components have a jump but the displacement field remains continuous. This is illustrated in Figure 1b. Since the displacement is continuous, the strain components in the plane tangential to the discontinuity surface must remain continuous as well, and only the out-of-plane components can have a jump. In physical terms, the band between the weak discontinuities corresponds to a damage process zone with an almost constant density of microdefects.

Finally, regularized models usually lead to a continuously differentiable displacement field, and the strain field remains continuous. Strain localization is manifested by high strains in a narrow band, with a continuous transition to much lower strains in the surrounding parts of the body. A typical strain profile of this type is shown in Figure 1c. In physical terms, this corresponds to a damage process zone with a continuously varying concentration of defects. 


\section{Regularized softening continua}

\subsection{Overview of generalized continuum models}

Fully regularized description of localized inelastic deformation can be achieved by a proper generalization of the underlying continuum theory. Generalized continua in the broad sense can be classified according to the following criteria:

1) Generalized kinematic relations (and the dual equilibrium equations).

a) Continua with microstructure, e.g., Cosserat-type continua or straingradient theories.

b) Continua with nonlocal strain, e.g., nonlocal elasticity.

2) Generalized constitutive equations.

a) Material models with gradients of internal variables (in some cases also with gradients of thermodynamic forces).

b) Material models with weighted spatial averages of internal variables (in some cases also of thermodynamic forces).

Here we focus on the second class of models, with enhancements on the level of the constitutive equations. Their advantage is that the kinematic and equilibrium equations remain standard, and the notions of stress and strain keep their usual meaning.

\subsection{Typical softening continuum model}

As a prototype of a softening continuum, we will consider the one-parameter isotropic damage model described by the stress-strain law

$$
\boldsymbol{\sigma}=(1-\omega) \boldsymbol{D}_{e}: \varepsilon
$$

damage law

$$
\omega=g(\kappa)
$$

and loading-unloading conditions

$$
f(\varepsilon, \kappa) \equiv \varepsilon_{\text {eq }}(\varepsilon)-\kappa \leq 0, \quad \dot{\kappa} \geq 0, \quad f(\varepsilon, \kappa) \dot{\kappa}=0
$$

in which $\boldsymbol{\sigma}$ is the stress tensor, $\boldsymbol{\varepsilon}$ is the strain tensor, $\boldsymbol{D}_{e}$ is the elastic material stiffness tensor, $\omega$ is the damage variable, $f$ is the damage loading function, $g$ is the damage evolution function, $\varepsilon_{\text {eq }}$ is a scalar measure of the strain level called the equivalent strain, and $\kappa$ is an internal variable that corresponds to the maximum level of equivalent strain ever reached in the previous history of the material. 
The choice of a specific expression for the equivalent strain directly affects the shape of the elastic domain in the strain space. For instance, one could define the equivalent strain as the scaled energy norm,

$$
\varepsilon_{\mathrm{eq}}=\sqrt{\frac{\varepsilon: D_{e}: \varepsilon}{E}}
$$

Square root and scaling by Young's modulus $E$ are used in order to transform the energy into a strain-like quantity, in the special case of uniaxial loading equal to the longitudinal strain.

Since damage in many materials propagates much more easily under tension than under compression, the specific expression for equivalent strain $\varepsilon_{\text {eq }}$ is usually designed such that the influence of tension is emphasized. As a first approximation, one may assume that compressive strains do not lead to any damage, and relate $\varepsilon_{\text {eq }}$ to positive strains only. For instance, Mazars (Mazars, 1984) defined the equivalent strain as

$$
\varepsilon_{\text {eq }}=\|\langle\varepsilon\rangle\|=\sqrt{\sum_{I=1}^{3}\left\langle\varepsilon_{I}\right\rangle^{2}}
$$

where $\varepsilon_{I}, I=1,2,3$, are the principal strains, and the brackets $\langle\ldots\rangle$ denote the positive part operator, defined by the relations $\langle\varepsilon\rangle=\varepsilon$ for $\varepsilon>0$ and $\langle\varepsilon\rangle=0$ for $\varepsilon \leq 0$.

As shown in (Jirásek, 2007), for sufficiently "fast" damage evolution (sufficiently high value of the derivative of damage function $g$ ) the localization tensor is singular for certain directions, the differential equations governing the mechanical problem lose ellipticity, and the damage model in the foregoing local form does not provide an objective description of the failure process.

\subsection{Integral-type nonlocal formulation}

Integral-type nonlocal models abandon the classical assumption of locality and admit that stress at a certain point depends not only on the state variables at that point but in general on the distribution of state variables over the whole body, or at least on their distribution in a finite neighborhood of the point under consideration. The first models of this type, proposed in the 1960s, aimed at improving the description of elastic wave dispersion in crystals. Nonlocal elasticity was further developed by Eringen, who extended it to nonlocal elastoplasticity (Eringen, 1981; Eringen, 1983) in the early 1980s. Subsequently it was found that certain nonlocal formulations can act as efficient localization limiters with a regularizing effect on problems with strain localization (Pijaudier-Cabot et al., 1987). Nonlocal formulations were elaborated for a wide spectrum of models, including softening plasticity (Bažant et al., 1988b; Strömberg et al., 1996), smeared crack models (Bažant et al., 1988a; Jirásek et al., 1997), or microplane models (Bažant et al., 1990; Ožbolt et al., 1996; Bažant et al., 2004). For a detailed account, the reader is referred to (Bažant et al., 2002). 
Generally speaking, the nonlocal approach consists in replacing a certain variable by its nonlocal counterpart obtained by weighted averaging over a spatial neighborhood of each point under consideration. If $f(\boldsymbol{x})$ is some "local" field in a domain $V$, the corresponding nonlocal field is defined as

$$
\bar{f}(\boldsymbol{x})=\int_{V} \alpha(\boldsymbol{x}, \boldsymbol{\xi}) f(\boldsymbol{\xi}) \mathrm{d} \boldsymbol{\xi}
$$

where $\alpha(\boldsymbol{x}, \boldsymbol{\xi})$ is a given nonlocal weight function. In an infinite body, the weight function depends only on the distance between the "source" point, $\boldsymbol{\xi}$, and the "target" point, $\boldsymbol{x}$. In the vicinity of a boundary, the weight function is usually rescaled such that the nonlocal operator does not alter a uniform field. This can be achieved by setting

$$
\alpha(\boldsymbol{x}, \boldsymbol{\xi})=\frac{\alpha_{0}(\|\boldsymbol{x}-\boldsymbol{\xi}\|)}{\int_{V} \alpha_{0}(\|\boldsymbol{x}-\boldsymbol{\zeta}\|) \mathrm{d} \boldsymbol{\zeta}}
$$

where $\alpha_{0}(r)$ is a monotonically decreasing nonnegative function of the distance $r=\|\boldsymbol{x}-\boldsymbol{\xi}\|$. In the one-dimensional setting, $x$ and $\xi$ are scalars and the domain of integration $V$ reduces to an interval.

The weight function is often taken as the Gauss distribution function (solid curve in Figure 2a)

$$
\alpha_{0}(r)=\exp \left(-\frac{r^{2}}{2 \ell^{2}}\right)
$$

where $\ell$ is a parameter reflecting the internal length of the nonlocal continuum. Another possible choice is the truncated quartic polynomial function (dashed curve in Figure 2a)

$$
\alpha_{0}(r)=\left\langle 1-\frac{r^{2}}{R^{2}}\right\rangle^{2}
$$

where $R$ is a parameter related to the internal length. Since $R$ corresponds to the largest distance of point $\boldsymbol{\xi}$ that affects the nonlocal average at point $\boldsymbol{x}$, it is called the interaction radius. The Gauss function [8] has an unbounded support, i.e., its interaction radius is $R=\infty$.

A suitable nonlocal damage formulation that restores well-posedness of the boundary value problem is obtained if damage is computed from the nonlocal equivalent strain. In the loading-unloading conditions [3], the local value $\varepsilon_{\text {eq }}$ is replaced by its weighted spatial average

$$
\bar{\varepsilon}_{\text {eq }}(\boldsymbol{x})=\int_{V} \alpha(\boldsymbol{x}, \boldsymbol{\xi}) \varepsilon_{\mathrm{eq}}(\boldsymbol{\xi}) \mathrm{d} \boldsymbol{\xi}
$$

According to the modified loading-unloading conditions

$$
\bar{\varepsilon}_{\text {eq }}-\kappa \leq 0, \quad \dot{\kappa} \geq 0, \quad\left(\bar{\varepsilon}_{\text {eq }}-\kappa\right) \dot{\kappa}=0
$$


(a)

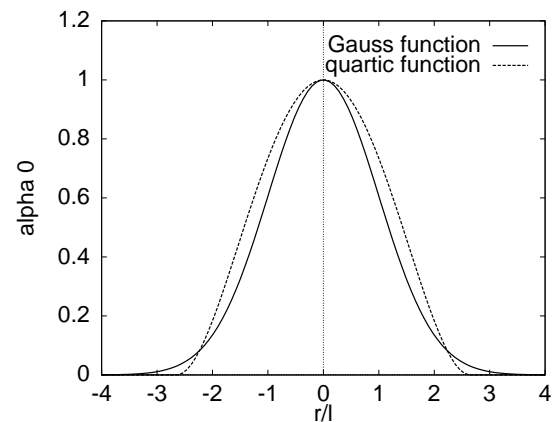

(c)

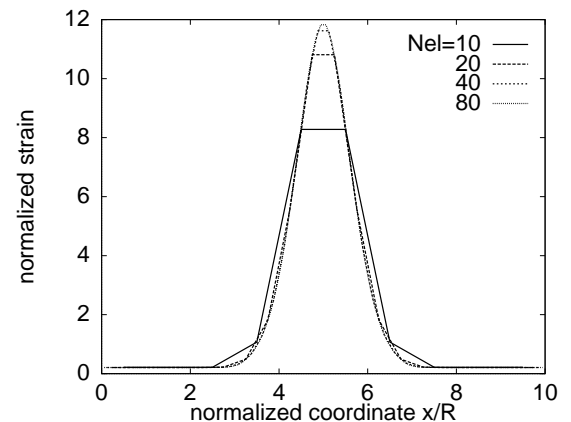

(b)

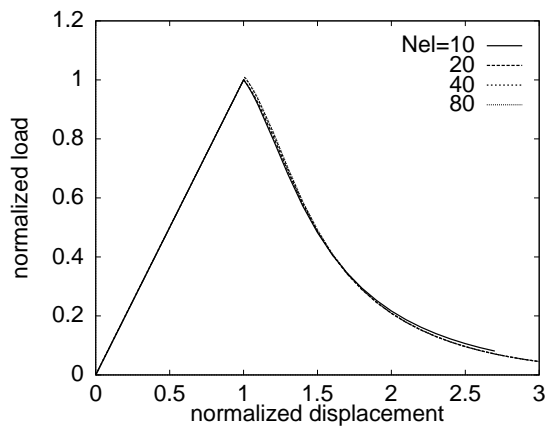

(d)

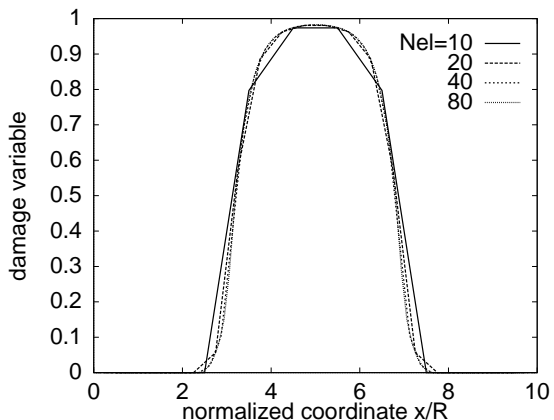

Figure 2. (a) Nonlocal weight functions $\alpha_{0}$, (b) convergence of load-displacement diagram, (c) convergence of strain profi le, (d) convergence of damage profi le; Nel= number of elements

the internal variable $\kappa$ has the meaning of the largest previously reached value of nonlocal equivalent strain $\bar{\varepsilon}_{\text {eq }}$. The corresponding damage variable evaluated from [2] is then substituted into the stress-strain equations [1]. It is important to note that the damage variable is evaluated from the nonlocal equivalent strain $\bar{\varepsilon}_{\text {eq }}$, but the strain $\varepsilon$ that appears in [1] explicitly is kept local. In the elastic range, the damage variable remains equal to zero, and the stress-strain relation is local.

Figure $2 \mathrm{~b}$ shows the load-displacement diagram for strain localization in a bar under uniaxial tension, calculated using a nonlocal damage model with the weight function [9] and with the exponential damage law

$$
\omega=g(\kappa)=1-\frac{\varepsilon_{0}}{\kappa} \exp \left(-\frac{\left\langle\kappa-\varepsilon_{0}\right\rangle}{\varepsilon_{\mathrm{f}}-\varepsilon_{0}}\right)
$$

where $\varepsilon_{0}$ is the limit elastic strain and $\varepsilon_{\mathrm{f}}$ is a parameter that controls softening. As the number of finite elements increases, the load-displacement curve rapidly converges to 
the exact solution. Convergence of strain and damage profiles generated by an applied displacement $u=2 u_{0}$ is documented in Figure 2c,d. In contrast to the local model, the process zone does not shrink to a single point as the mesh is refined. Its size is controled by the interaction radius $R$, considered as a material parameter.

\subsection{Explicit gradient formulation}

Gradient models can be considered as the differential counterpart of integral nonlocal formulations. Instead of dealing with integrals that represent spatial interactions, we can take the microstructure into account by incorporating the influence of gradients (of the first or higher order) of internal variables into the constitutive relations. The most popular example is the gradient-dependent plasticity theory that evolved from the original ideas of Aifantis (Aifantis, 1984). If a similar approach is used in damage mechanics, damage is assumed to be driven not only by the (local) equivalent strain $\varepsilon_{\text {eq }}$ but also by its Laplacean, $\nabla^{2} \varepsilon_{\text {eq }}$, which represents in a generalized sense the "curvature" of the strain distribution. In the simplest case, $\varepsilon_{\text {eq }}$ is replaced in the loading-unloading conditions [3] by the quantity

$$
\bar{\varepsilon}_{\mathrm{eq}}=\varepsilon_{\mathrm{eq}}+\ell^{2} \nabla^{2} \varepsilon_{\mathrm{eq}}
$$

where $\ell$ is a material parameter with the dimension of length. The damage-driving quantity $\bar{\varepsilon}_{\text {eq }}$ can be considered as a specific type of nonlocal equivalent strain, which is now constructed by applying a differential operator on the local equivalent strain, rather than by applying the integral operator according to [10]. Therefore, the gradient damage model based on [13] is considered as weakly nonlocal. ${ }^{1}$

As long as the strain distribution remains uniform (such as in a uniaxial tensile test before localization), the equivalent strain is also uniform, its Laplacean vanishes, and the model response is exactly the same as for the local formulation. After the onset of localization, the higher-order term is activated and prevents localization of damage in a set of zero measure.

It is instructive to discuss how the gradient term limits localization. Around the point that experiences the largest strain, the curvature of the strain profile is negative, and due to the Laplacean term in [13] the nonlocal equivalent strain is smaller than the local one. If the softening zone were too narrow, the negative curvature of the strain profile around its peak would have a large magnitude, and the damage evolution would be very slow. This would slow down the strain growth in the central part of the localized zone and accelerate the growth in the adjacent regions, so the localization zone would expand. The minimum size of this zone is controled by the length parameter $\ell$, as will be shown in Section 3.3.

1. For strongly nonlocal models, the value of the nonlocal quantity at a certain point depends on the distribution of the corresponding local quantity in the entire body or at least in a fi nite neighborhood of that point, while for weakly nonlocal models it can be computed from the distribution of the local quantity in an arbitrarily small neighborhood. 


\subsection{Implicit gradient formulation}

Due to the presence of second derivatives of internal variables, the numerical implementation of explicit gradient models is not easy. The simplest version of the explicit gradient damage model suffers by certain deficiencies, which will be described in more detail in (Simone, 2007). These problems can be overcome by the implicit gradient damage formulation (Peerlings et al., 1996), which defines the nonlocal variable indirectly as the solution of a Helmholtz-type differential equation

$$
\bar{\varepsilon}_{\mathrm{eq}}-\ell^{2} \nabla^{2} \bar{\varepsilon}_{\mathrm{eq}}=\varepsilon_{\mathrm{eq}}
$$

with the homogeneous Neumann boundary condition $\boldsymbol{n} \cdot \boldsymbol{\nabla} \bar{\varepsilon}_{\mathrm{eq}}=0$ imposed on the entire physical boundary $S$ of the body $V$.

The solution $\bar{\varepsilon}$ of the above boundary value problem can be expressed in the form of an averaging formula similar to [10] with the weight function $\alpha(\boldsymbol{x}, \boldsymbol{\xi})$ replaced by the Green function of the boundary value problem. For instance, for an infinite one-dimensional domain, the Green function of the Helmholtz equation [14] is given by

$$
G(x, \xi)=\frac{1}{2 \ell} \exp \left(-\frac{|x-\xi|}{\ell}\right)
$$

So the implicit gradient models are equivalent to integral-type nonlocal models with special nonlocal weight functions. Despite this formal equivalence, their numerical implementation is quite different (Peerlings et al., 1996).

\section{One-dimensional localization analysis}

To shed more light on the regularizing effect of various nonlocal damage formulations, we will perform a simple one-dimensional analysis of bifurcations from a uniform state under uniaxial tension. This is just an academic example, but it is instructive because it can be solved analytically or semi-analytically and the results indicate which parameters influence the size of the localized zone.

\subsection{General considerations}

We consider a perfectly homogeneous bar with constant cross section, subjected to increasing total elongation. No body or inertia forces are taken into account, and so the stress state is uniform. The problem always admits a solution with uniform strain. The main questions to be addressed here are under which conditions the strain distribution can become nonuniform and how such nonuniform solutions look like. We analyze the state at incipient loss of strain uniformity, when the current strain is still uniform but the strain rate is not. 
The rate form of the stress-strain law [1] reduced to one spatial dimension and combined with the rate form of the damage law [2] reads

$$
\dot{\sigma}=(1-\omega) E \dot{\varepsilon}-E \varepsilon \dot{\omega}=E_{\mathrm{u}} \dot{\varepsilon}-E \varepsilon g^{\prime} \dot{\kappa}
$$

where $E_{\mathrm{u}}=(1-\omega) E$ is the unloading (secant) modulus and $g^{\prime}=\mathrm{d} g / \mathrm{d} \kappa$ is the derivative of the damage function $g$.

Consider first the local damage model. Up to the current state, the material has experienced no unloading and there is no difference between the damage-driving variable $\kappa$ and the longitudinal strain $\varepsilon$. But the rate $\dot{\kappa}$ is equal to the strain rate $\dot{\varepsilon}$ only if the strain is growing, otherwise we have $\dot{\kappa}=0$. Both cases are covered by the relation $\dot{\kappa}=\langle\dot{\varepsilon}\rangle$. In the damage zone, characterized by $\dot{\varepsilon}>0$, expression [16] for the stress rate can be written as $\dot{\sigma}=E_{\mathrm{ed}} \dot{\varepsilon}$ where $E_{\mathrm{ed}}=E_{\mathrm{u}}-E \varepsilon g^{\prime}$ is the elasto-damage (tangent) modulus. Replacing $-E \varepsilon g^{\prime}$ by $E_{\text {ed }}-E_{\mathrm{u}}$, we can rewrite [16] as

$$
\dot{\sigma}=E_{\mathrm{u}} \dot{\varepsilon}+\left(E_{\mathrm{ed}}-E_{\mathrm{u}}\right) \dot{\kappa}
$$

Let us now turn attention to nonlocal models. For all nonlocal damage formulations presented in the preceding section, damage is driven by the nonlocal equivalent strain $\bar{\varepsilon}$, and the rate of the internal variable $\kappa$ at the onset of localization can be expressed as $\dot{\kappa}=\langle\dot{\bar{\varepsilon}}\rangle$. Relation [17] written for the entire bar provides an equation that governs the distribution of the strain rate. This equation, in general written as

$$
E_{\mathrm{u}} \dot{\varepsilon}(x)+\left(E_{\mathrm{ed}}-E_{\mathrm{u}}\right)\langle\dot{\bar{\varepsilon}}(x)\rangle=\dot{\sigma}
$$

has an integral or differential character, depending on the specific definition of the nonlocal operator. Note that the current values of the moduli $E_{\mathrm{u}}$ and $E_{\text {ed }}$ are taken as constants because at the onset of localization the current state is still uniform, and that the stress rate $\dot{\sigma}$ is also independent of the spatial coordinate-its uniformity follows from the equilibrium equation. Depending on the sign of the stress rate and of the tangent modulus, equation [18] has uniform solutions $\dot{\varepsilon}(x)=\dot{\sigma} / E_{\text {ed }}>0$ or $\dot{\varepsilon}(x)=$ $\dot{\sigma} / E_{\mathrm{u}}<0$ that correspond to uniform damage growth or to uniform elastic unloading, respectively. We will now look for nonuniform solutions admitted by various nonlocal formulations.

For a given ratio $E_{\mathrm{ed}} / E_{\mathrm{u}}$, the exact width and shape of the strain rate profile can be found numerically. This could be done by the finite element method, but the bifurcation would have to be triggered by an initial imperfection and the results (especially the shape of the profile around the imperfection) might be sensitive to the magnitude and extent of that imperfection. It is therefore preferable to solve equation [18] directly, with $\dot{\sigma}$ considered as a given (negative) constant. The most interesting solution is that for which the growth of damage localizes into an interval $I_{\mathrm{d}}$ of finite length $L_{\mathrm{d}}$, surrounded by elastically unloading material with constant damage. The origin of the coordinate system will be placed in the center of the localized damage zone, which means that $I_{\mathrm{d}}$ will be considered as the interval $\left(-L_{\mathrm{d}} / 2, L_{\mathrm{d}} / 2\right)$.

For simplicity, we assume that the total bar length $L$ is sufficiently large, so that $I_{\mathrm{d}}$ is contained in the interval $(-L / 2, L / 2)$ representing the entire bar. Outside $I_{\mathrm{d}}$, 
the nonlocal strain rate is nonpositive and [18] reduces to $E_{\mathrm{u}} \dot{\varepsilon}(x)=\dot{\sigma}$, from which $\dot{\varepsilon}(x)=\dot{\sigma} / E_{\mathrm{u}}$ for $x \notin I_{\mathrm{d}}$. It is convenient to introduce a new unknown function $\dot{e}(x)=\dot{\varepsilon}(x)-\dot{\sigma} / E_{\mathrm{u}}$ (which is nonvanishing only in $I_{\mathrm{d}}$ ) and rewrite [18] as

$$
\frac{E_{\mathrm{u}}}{E_{\mathrm{u}}-E_{\mathrm{ed}}} \dot{e}(x)-\dot{\bar{e}}(x)=\frac{\dot{\sigma}}{E_{\mathrm{u}}} \quad \text { for } x \in I_{\mathrm{d}}
$$

The nonstandard feature is that the interval $I_{d}$ on which [19] is to be solved is not known in advance-it must be found from the loading-unloading conditions, which require that $I_{\mathrm{d}}=\{x \mid \dot{\bar{\varepsilon}}(x)>0\}$, i.e., in terms of the new unknown function, that $I_{\mathrm{d}}=\left\{x \mid \dot{\bar{e}}(x)>-\dot{\sigma} / E_{\mathrm{u}}\right\}$. Physically, $\dot{e}$ can be considered as the damage strain rate or inelastic strain rate, because the rate form of the stress-strain law can be presented as $\dot{\sigma}=E_{\mathrm{u}}(\dot{\varepsilon}-\dot{e})$.

\subsection{Integral formulation}

For the integral-type nonlocal damage model with averaging of equivalent strain, [19] reads

$$
\frac{E_{\mathrm{u}}}{E_{\mathrm{u}}-E_{\mathrm{ed}}} \dot{e}(x)-\int_{-L_{\mathrm{d}} / 2}^{L_{\mathrm{d}} / 2} \alpha(x, \xi) \dot{e}(\xi) \mathrm{d} \xi=\frac{\dot{\sigma}}{E_{\mathrm{u}}} \quad \text { for } x \in I_{\mathrm{d}}
$$

This is a Fredholm integral equation of the second kind with a constant right-hand side. Due to its integral definition, the nonlocal strain rate $\dot{\bar{\varepsilon}}$ is continuous even if the local strain rate $\dot{\varepsilon}$ is not. Since $\dot{\bar{\varepsilon}}$ is positive inside the damage zone $I_{\mathrm{d}}$ and nonpositive outside, it must vanish on the boundary of $I_{\mathrm{d}}$, i.e., at points $x=-L_{\mathrm{d}} / 2$ and $x=$ $L_{\mathrm{d}} / 2$. Consequently, the value of $\dot{\bar{e}}$ at these points must be $-\dot{\sigma} / E_{\mathrm{u}}$. This is the condition from which the actual size of the damage zone $L_{\mathrm{d}}$ can be determined. The problem is formally identical with that arising in localization analysis of the VermeerBrinkgreve nonlocal plasticity model (Vermeer et al., 1994; Jirásek et al., 2003), and it can be solved using the iterated collocation scheme proposed in (Planas et al., 1996). The numerical results are represented by the isolated points in Figure 3.

For small sizes of the damage zone, an analytical approximation can be constructed by expanding the weight function into the Taylor series around the origin. For the truncated quartic weight function [9], asymptotic analysis leads to the formula

$$
L_{\mathrm{d}} \approx\left(\frac{-3.2 R^{3} E_{\mathrm{u}}}{E_{\mathrm{ed}}}\right)^{1 / 3}=1.4736 R\left(-\frac{E_{\mathrm{u}}}{E_{\mathrm{ed}}}\right)^{1 / 3}
$$

Figure $3 \mathrm{a}$ shows that this approximation is accurate only for ratios $-E_{\mathrm{ed}} / E_{\mathrm{u}}$ larger than about 2. An explicit formula valid for small values of this ratio was derived in (Patzák et al., 2003) in the form

$$
L_{\mathrm{d}} \approx \pi R \sqrt{-\frac{2 E_{\mathrm{u}}}{7 E_{\mathrm{ed}}}}=1.6793 R\left(-\frac{E_{\mathrm{u}}}{E_{\mathrm{ed}}}\right)^{1 / 2}
$$


(a)

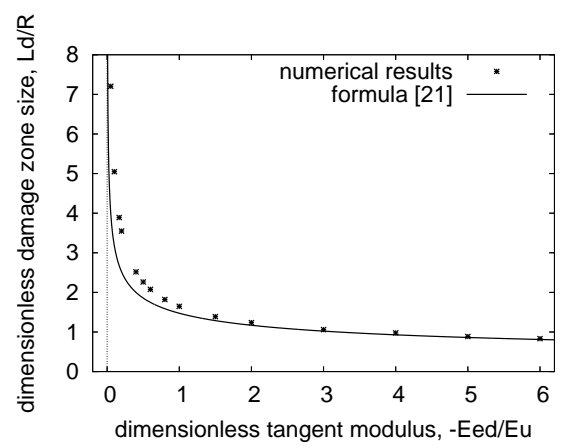

(b)

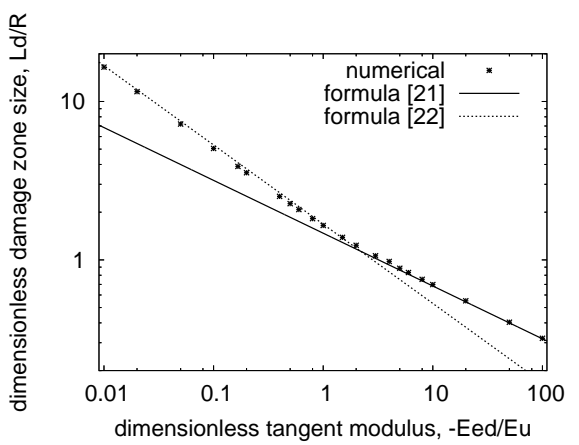

Figure 3. Size of the localized damage zone as a function of the ratio between the tangent and unloading modulus: (a) natural scale, (b) logaritmic scale

To get a better idea about the dependence of the damage zone size on the model parameters in a wide range, we plot the results in a logarithmic scale. Figure $3 \mathrm{~b}$ shows that the combination of formulae [21] and [22] provides a very good estimate of $L_{\mathrm{d}}$ over the entire range of ratios $E_{\mathrm{ed}} / E_{\mathrm{u}}$. Formula [21] should be applied if $-E_{\mathrm{ed}} / E_{\mathrm{u}} \geq 2.19$ and formula [22] if $-E_{\mathrm{ed}} / E_{\mathrm{u}} \leq 2.19$. So the initial width of the localized damage zone can be directly determined from the model parameters $E_{\text {ed }}, E_{\mathrm{u}}$ and $R$.

Let us emphasize that the foregoing analysis refers only to the bifurcation from a uniform state, which occurs at the onset of softening, provided that the bar is sufficiently large. For example, for the damage evolution law [12] that corresponds to exponential softening, the peak of the local stress-strain diagram coincides with the limit elastic state (for which the current value of damage is still equal to zero), and so $E_{\mathrm{u}}$ should be interpreted as the elastic modulus $E$ and $E_{\text {ed }}$ as the initial slope of the softening branch of the stress-strain diagram, $E_{\text {ed }}=-E \varepsilon_{0} /\left(\varepsilon_{\mathrm{f}}-\varepsilon_{0}\right)$. The ratio $E_{\text {ed }} / E_{\mathrm{u}}=-\varepsilon_{0} /\left(\varepsilon_{\mathrm{f}}-\varepsilon_{0}\right)=-1 /\left(\varepsilon_{\mathrm{f}} / \varepsilon_{0}-1\right)$ depends only on the ratio $\varepsilon_{\mathrm{f}} / \varepsilon_{0}$, which is a dimensionless measure of the (local) ductility. For a given ratio $\varepsilon_{\mathrm{f}} / \varepsilon_{0}$, the initial size of the localized damage zone is according to [21]-[22] directly proportional to the nonlocal interaction radius $R$.

\subsection{Explicit gradient formulation}

For the explicit gradient damage model, the nonlocal strain that drives damage is given by [13], and its rate is $\dot{\bar{\varepsilon}}=\dot{\varepsilon}+\ell^{2} \dot{\varepsilon}^{\prime \prime}$. The rate of nonlocal inelastic strain is given by a similar expression $\dot{\bar{e}}=\dot{e}+\ell^{2} \dot{e}^{\prime \prime}$, because $\dot{\varepsilon}$ and $\dot{e}$ differ only by a constant, $\dot{\sigma} / E_{\mathrm{u}}$. 
Substituting this expression into [19], we find that the inelastic strain rate inside the localized damage zone $I_{\mathrm{d}}$ must satisfy the differential equation

$$
\frac{E_{\mathrm{ed}}}{E_{\mathrm{u}}-E_{\mathrm{ed}}} \dot{e}(x)-\ell^{2} \dot{e}^{\prime \prime}(x)=\frac{\dot{\sigma}}{E_{\mathrm{u}}} \quad \text { for } x \in I_{\mathrm{d}}
$$

and outside the damage zone we have $\dot{e}(x)=0$. Rigorous mathematical description of the continuity conditions on the boundary of the damage zone is in general not trivial, but in the present simple case of perfectly uniform bar it can be shown that the inelastic strain rate must be continuously differentiable in the entire domain, and since it vanishes outside $I_{\mathrm{d}}$, its value and derivative on the boundary of $I_{\mathrm{d}}$ must also vanish. Therefore, we solve equation [23] with conditions $\dot{e}\left( \pm L_{\mathrm{d}} / 2\right)=0$ and $\dot{e}^{\prime}\left( \pm L_{\mathrm{d}} / 2\right)=0$ applied at points $x=-L_{\mathrm{d}} / 2$ and $x=L_{\mathrm{d}} / 2$, which represent the boundary of the damage zone. Straightforward analysis leads to the conclusion that for $E_{\text {ed }} \geq 0$ this boundary value problem admits only the trivial solution $\dot{e}(x)=0$, while for $E_{\text {ed }}<0$ there exist other solutions. The most interesting one is that which localizes into the shortest possible interval of length

$$
L_{\mathrm{d}}=2 \pi \ell \sqrt{1-\frac{E_{\mathrm{u}}}{E_{\mathrm{ed}}}}
$$

and has the form

$$
\dot{e}(x)=\frac{\dot{\sigma}\left(E_{\mathrm{u}}-E_{\mathrm{ed}}\right)}{E_{\mathrm{u}} E_{\mathrm{ed}}}\left(1+\cos \frac{2 \pi x}{L_{\mathrm{d}}}\right)
$$

The distribution of the strain rate $\dot{\varepsilon}=\dot{e}+\dot{\sigma} / E_{\mathrm{u}}$ for such localized solution is plotted in Figure $4 \mathrm{a}$, along with the nonlocal strain rate. These distributions have been constructed for the specific case $E_{\mathrm{ed}}=-E_{\mathrm{u}}$, in which the size of the damage zone is $L_{\mathrm{d}}=2 \sqrt{2} \pi \ell=8.886 \ell$. The values on the vertical axis are normalized by $-\dot{\sigma} / E_{\mathrm{u}}$. Note that the nonlocal strain rate has a jump at the boundary of the damage zone, because the second derivative of the local strain rate is not continuous.

According to [24], the size $L_{\mathrm{d}}$ of the localized zone is directly proportional to the characteristic length $\ell$, but it also depends on the ratio between the tangent and unloading moduli. For "mild softening" with small negative values of the tangent modulus $E_{\text {ed }}$, the size of the damage zone is much larger than the characteristic length, while for "abrupt softening" with high negative $E_{\text {ed }}$ it is close to the minimum possible value $2 \pi \ell$. So it can be expected that for damage laws that lead to a sharp peak of the local stress-strain diagram, such as the exponential law [12], localization will occur right at peak stress, provided that the total length of the bar is larger than the size of the localized zone predicted by [24]. For damage laws that lead to a continuous evolution of the tangent modulus, the localized zone will develop gradually and its size is expected to shrink as the negative ratio $E_{\text {ed }} / E_{\mathrm{u}}$ increases in magnitude. 
(a)

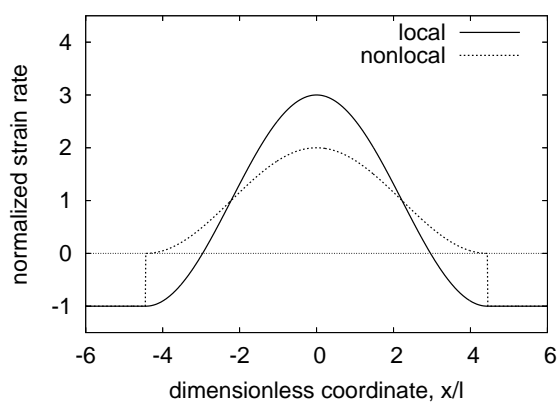

(b)

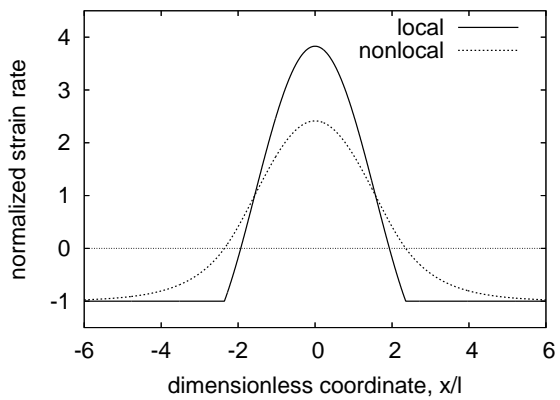

Figure 4. Distribution of local and nonlocal strain rate according to the (a) explicit, (b) implicit gradient damage model

\subsection{Implicit gradient damage formulation}

For the implicit gradient damage model, the nonlocal strain $\bar{\varepsilon}$ driving damage is defined implicitly by the differential equation [14], and so we cannot easily eliminate it from [18]. Therefore, we consider the nonlocal strain rate $\dot{\bar{\varepsilon}}$ as the primary unknown and eliminate the rate of local strain $\dot{\varepsilon}$. Combining [14] with [18] we find out that the nonlocal strain rate is governed by a differential equation that has a different form inside and outside the damage zone:

$$
\begin{aligned}
E_{\mathrm{ed}} \dot{\bar{\varepsilon}}(x)-E_{\mathrm{u}} \ell^{2} \dot{\bar{\varepsilon}}^{\prime \prime}(x) & =\dot{\sigma} & & \text { for } x \in I_{\mathrm{d}} \\
E_{\mathrm{u}} \dot{\bar{\varepsilon}}(x)-E_{\mathrm{u}} \ell^{2} \dot{\bar{\varepsilon}}^{\prime \prime}(x) & =\dot{\sigma} & & \text { for } x \notin I_{\mathrm{d}}
\end{aligned}
$$

To satisfy the loading-unloading conditions, the solution $\dot{\bar{\varepsilon}}$ should be nonnegative in the damage zone and nonpositive outside and thus, due to continuity requirements, it must vanish on the boundary of the damage zone. So we solve equations [26][27] with conditions $\dot{\bar{\varepsilon}}\left( \pm L_{\mathrm{d}} / 2\right)=0$ and with imposed continuous differentiability at points $x=-L_{\mathrm{d}} / 2$ and $x=L_{\mathrm{d}} / 2$. After straightforward mathematical analysis, we come to the conclusion that a nonuniform solution exists only for $E_{\text {ed }}<0$ and that it has the form

$$
\dot{\bar{\varepsilon}}(x)= \begin{cases}\frac{\dot{\sigma}}{E_{\mathrm{ed}}}\left(1-\frac{\cos \sqrt{-E_{\mathrm{ed}} / E_{\mathrm{u}}} x / \ell}{\cos \sqrt{-E_{\mathrm{ed}} / E_{\mathrm{u}}} L_{\mathrm{d}} / 2 \ell}\right) & \text { for }|x| \leq L_{\mathrm{d}} / 2 \\ \frac{\dot{\sigma}}{E_{\mathrm{u}}}\left[1-\exp \left(\frac{L_{\mathrm{d}}-2|x|}{2 \ell}\right)\right] & \text { for }|x|>L_{\mathrm{d}} / 2\end{cases}
$$


where the size of the damage zone is

$$
L_{\mathrm{d}}=2 \ell \sqrt{-\frac{E_{\mathrm{u}}}{E_{\mathrm{ed}}}}\left(\pi-\arctan \sqrt{-\frac{E_{\mathrm{ed}}}{E_{\mathrm{u}}}}\right)
$$

The corresponding local strain rate is then easily evaluated as $\dot{\varepsilon}=\dot{\bar{\varepsilon}}-\ell^{2} \dot{\bar{\varepsilon}}^{\prime \prime}$. The resulting profiles of local and nonlocal strain rate are plotted in Figure $4 \mathrm{~b}$, again for the specific case with $E_{\mathrm{ed}}=-E_{\mathrm{u}}$. The size of the damage zone according to [29] is $L_{\mathrm{d}}=1.5 \pi \ell=4.712 \ell$. Note that, in contrast to the explicit gradient formulation with strain rates plotted in Figure $4 a$, the nonlocal strain rate is now continuously differentiable but the local strain rate is only continuous and its derivative has a jump on the boundary of the damage zone.

For simplicity, we have presented here a solution valid on an infinite domain. For a bar of a finite length $L$, the homogeneous Neumann boundary conditions $\dot{\bar{\varepsilon}}^{\prime}=0$ must be imposed at $x=-L / 2$ and $x=L / 2$. The result is then more algebraically involved and depends on the bar length $L$, but the main characteristics are the same. The size of the localized damage zone $L_{\mathrm{d}}$ can be arbitrarily large for sufficiently small magnitude of the tangent modulus $E_{\text {ed }}$ (and sufficiently large bar length $L$ ), and it can be arbitrarily small for sufficiently large magnitude of $E_{\text {ed }}$. In contrast to the explicit gradient formulation, there is no lower limit on $L_{\mathrm{d}}$, and it can be expected that the active part of the damage zone shrinks during the softening process. This is indeed confirmed by numerical simulations.

In this section, we have investigated the bifurcations from a uniform state for three nonlocal damage formulations. We have found certain similarities but also some differences. The results are compared in Figure 5, which shows the dependence of the damage zone size on the tangent modulus. Each of the nonlocal enhancements introduces a new parameter that sets the internal length scale of the material-the integral formulation uses the nonlocal interaction radius $R$, and the gradient formulations multiply the second gradient of local or nonlocal strain by the square of an internal length parameter $\ell$. For small magnitudes of the negative tangent modulus $E_{\text {ed }}$, i.e., for very ductile local behavior, the size of the localized damage zone tends to infinity for all three formulations, proportionally to $\sqrt{-E_{\mathrm{u}} / E_{\mathrm{ed}}}$. If we set $R=\ell \sqrt{14}$, the dependence of the damage zone size on the tangent modulus tends to the same asymptotic limit $L_{\mathrm{d}} \approx 2 \pi \ell \sqrt{-E_{\mathrm{u}} / E_{\mathrm{ed}}}$ for all three formulations; see Figure 5. On the other hand, for very brittle local behavior with a high magnitude of the negative tangent modulus, the size of the damage zone tends to zero for the integral formulation and for the implicit gradient formulation, while for the explicit gradient formulation it tends to a finite limit $2 \pi \ell$. This indicates that we can expect differences in the evolution of the active part of the damage zone during the softening process. For the weakly nonlocal explicit gradient damage model, spurious expansion of the damage zone at late stages of softening is sometimes observed, and so the strongly nonlocal formulations (integral or implicit gradient) should be preferred. 


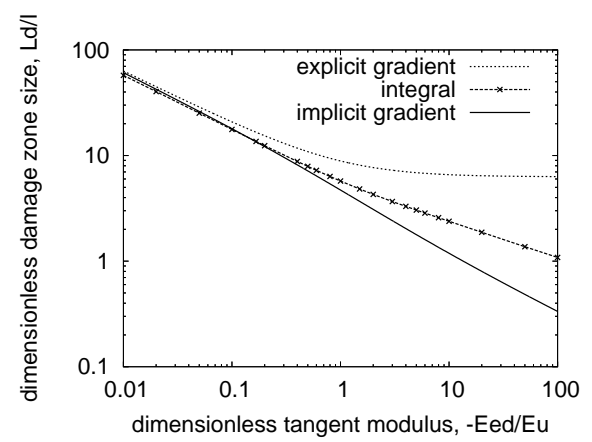

Figure 5. Dependence of the dimensionless size of the damage zone, $L_{\mathrm{d}} / \ell$, on the ratio between the tangent and unloading modulus, $-E_{\mathrm{ed}} / E_{\mathrm{u}}$, for different nonlocal formulations

\section{Comparison of various integral-type nonlocal formulations}

\subsection{Choice of nonlocal variable}

From a purely phenomenological point of view, the choice of the variable to be averaged remains to some extent arbitrary, provided that a few basic requirements are satisfied. First of all, we usually want the enriched model to exactly agree with the standard local elastic continuum as long as the material behavior remains in the elastic range. For this reason, it is not possible to simply replace the local strain by nonlocal strain and apply the usual constitutive law. Except for the case of homogeneous strain, nonlocal strain differs from the local one and the model behavior would be altered already in the elastic range. Second, the model should give a realistic response in simple loading situations such as uniaxial tension. This aspect was studied in (Jirásek, 1998), where it was shown that e.g. averaging of the damage parameter leads to spurious locking effects at later stages of softening.

Figure 6 shows the load-displacement diagrams computed for a uniform bar under uniaxial tension using nonlocal formulations with different choices of the averaged variable. The curves in Figure 6a correspond to models which apply the nonlocal operator on the equivalent strain $(\omega(\bar{\varepsilon}))$, on the damage energy release rate $(\bar{Y})$, or on the compliance variable $(\bar{\gamma})$. The damage energy release rate is defined as $Y=\varepsilon: D_{e}: \varepsilon / 2$ and it is proportional to the square of the energy-based equivalent strain [4]. The compliance variable $\gamma=\omega /(1-\omega)$ is directly related to the damage variable $\omega$ and it grows from zero to infinity as the damage evolves. For all these nonlocal formulations, the stress transmitted by the bar tends to zero as the applied displacement tends to infinity, and so the softening process is properly described until complete failure. In contrast to that, the load-displacement curves in Figure $6 \mathrm{~b}$ exhibit a pathological behavior - the stress transmitted by the bar at later stages of loading is too high and for some formulations even tends to a finite limit as the applied displace- 
(a)

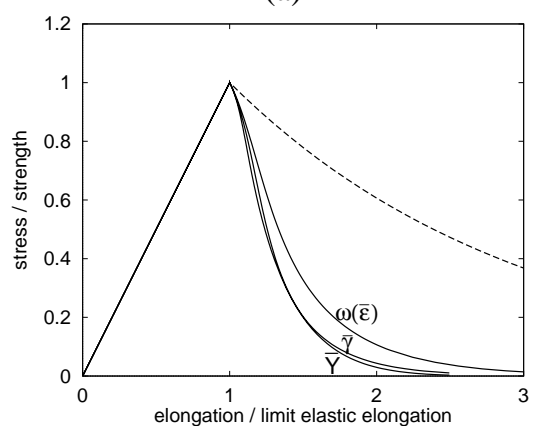

(b)

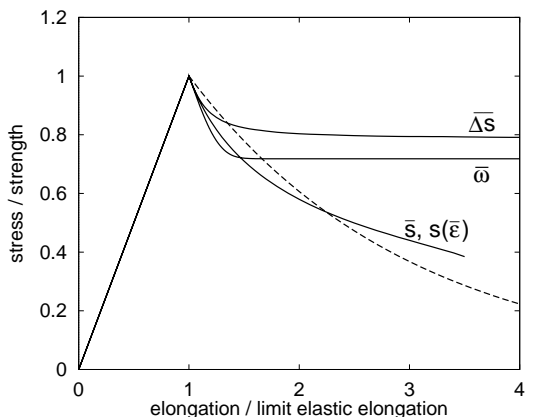

Figure 6. Comparison of postpeak load-displacement curves of a tensioned bar calculated for nonlocal damage with averaging applied to different variables: (a) nonlocking formulations $\omega(\bar{\varepsilon}), \bar{Y}$ and $\bar{\gamma} ;(b)$ locking formulations $\bar{\omega}, \bar{s}, \bar{\Delta} s$ and $s(\bar{\varepsilon})$

ment tends to infinity. This non-physical stress locking is typically accompanied by spurious expansion of the damage zone at late stages of loading and its source is in an inappropriate choice of the averaged variable. Formulations $\bar{\omega}, \bar{s}$ and $\bar{\Delta} s$ are based on nonlocal averaging of the damage variable, inelastic stress and inelastic stress increment, respectively, while formulation $s(\bar{\varepsilon})$ computes the inelastic stress from the nonlocal strain. For more details, the reader is referred to (Jirásek, 1998).

\subsection{Treatment of boundaries}

One of the drawbacks of gradient formulations is that they require special boundary conditions, which usually do not have a direct physical meaning and their choice is somewhat ambiguous. Integral formulation do not require boundary conditions in the traditional sense, but the influence of the physical boundaries still needs to be considered. If the nonlocal weight function were defined simply as $\alpha(\boldsymbol{x}, \boldsymbol{\xi})=\alpha_{0}(\|\boldsymbol{x}-\boldsymbol{\xi}\|)$ at all points $x$, even at those that are close to the boundary of the body $V$ under consideration, spurious boundary effects would arise. The reason is that the domain of influence that contributes to the nonlocal average evaluated at a point near the boundary is smaller than for "regular" points that are sufficiently far from the boundary. For instance, in a finite body under uniform strain, the local equivalent strain is everywhere the same but, if the weight function depends only on the distance, the nonlocal equivalent strain has lower values in boundary layers of thickness $R$ than in the interior part of the body. Consequently, damage evolution in the boundary layers is delayed and these layers appear as stronger than the interior part of the body. To avoid such an artificial strengthening of the boundary layer, a suitable modification of the averaging operator is necessary. However, the exact form of this modification is not known, and one needs to be guided by intuition, or perhaps by some micromechanical considerations. 
A standard requirement imposed on the nonlocal averaging operator is the socalled reproducibility of order zero, which means that a constant field (polynomial of order zero) should not be modified by nonlocal averaging. This requirement leads to the normalizing condition

$$
\int_{V} \alpha(\boldsymbol{x}, \boldsymbol{\xi}) \mathrm{d} \boldsymbol{\xi}=1
$$

and ensures that, under uniform strain, the damage threshold is reached by all points of the body simultaneously.

Condition [30] is easily satisfied if the distance-dependent weight function is rescaled according to [7]. This is the most frequently used modification of the averaging operator near the boundary. Its drawback is that symmetry with respect to the arguments $\boldsymbol{x}$ and $\boldsymbol{\xi}$ of the weight function $\alpha$ is lost.

In certain types of nonlocal theories it is desirable to work with a symmetric weight function. (Polizzotto, 2002) and (Borino et al., 2002) proposed a nonlocal averaging formula

$$
\bar{f}(\boldsymbol{x})=\frac{1}{A_{\infty}} \int_{V} \alpha_{0}(\|\boldsymbol{x}-\boldsymbol{\xi}\|) f(\boldsymbol{\xi}) \mathrm{d} \boldsymbol{\xi}+\left(1-\frac{A(\boldsymbol{x})}{A_{\infty}}\right) f(\boldsymbol{x})
$$

in which

$$
A(\boldsymbol{x})=\int_{V} \alpha_{0}(\|\boldsymbol{x}-\boldsymbol{\xi}\|) \mathrm{d} \boldsymbol{\xi}
$$

and $A_{\infty}$ is the value of $A(\boldsymbol{x})$ in an unbounded medium (or at points $\boldsymbol{x}$ sufficiently far from the boundary). This modification preserves symmetry and satisfies the condition of reproducibility of degree zero. It also has an attractive physical interpretation, because formula [31] can be rewritten as

$$
\bar{f}(\boldsymbol{x})=f(\boldsymbol{x})+\frac{1}{A_{\infty}} \int_{V} \alpha_{0}(\|\boldsymbol{x}-\boldsymbol{\xi}\|)[f(\boldsymbol{\xi})-f(\boldsymbol{x})] \mathrm{d} \boldsymbol{\xi}
$$

which means that the spatial averaging with a distance-dependent weight function is in fact applied to the difference between the local values at the source point and the receiver point.

It is interesting to note that another averaging formula satisfying symmetry and reproducibility of order zero can be derived from the implicit gradient formulation. It has already been explained that the implicit gradient definition of the nonlocal field can be rewritten in the integral format, with the Green function of the Helmholtztype boundary value problem used as the weight function in the nonlocal averaging operator. Since the original differential operator is self-adjoint, the Green function is symmetric. For instance, consider the one-dimensional problem

$$
\bar{f}(x)-\ell^{2} \bar{f}^{\prime \prime}(x)=f(x)
$$


(a)

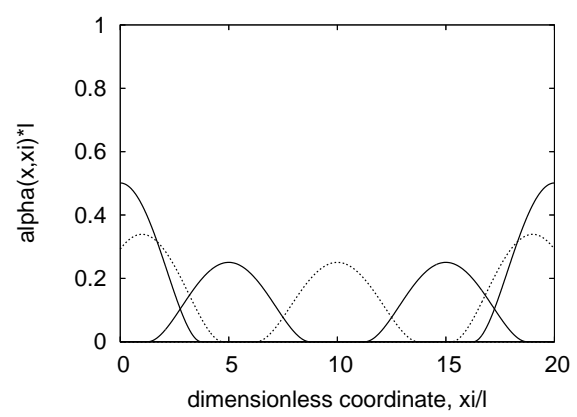

(b)

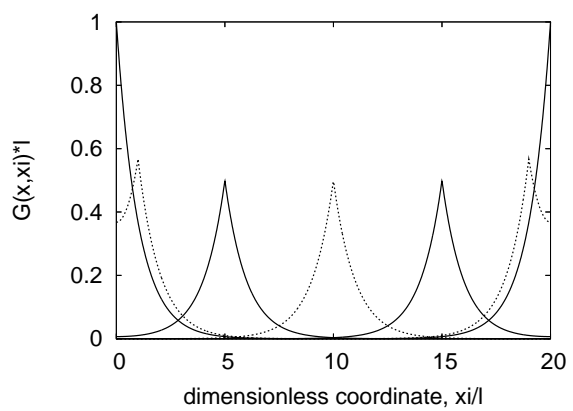

Figure 7. Modifi ed shapes of nonlocal weight functions near the boundaries: (a) rescaling, (b) implicit gradient approach

solved on the finite interval $(0, L)$ with boundary conditions

$$
\bar{f}^{\prime}(0)=0, \quad \bar{f}^{\prime}(L)=0
$$

The corresponding Green function

$$
G(x, \xi)= \begin{cases}\frac{\cosh \frac{L-\xi}{\ell}}{\ell \sinh \frac{L}{\ell}} \cosh \frac{x}{\ell} & \text { for } 0 \leq x \leq \xi \\ \frac{\cosh \frac{\xi}{\ell}}{\ell \sinh \frac{L}{\ell}} \cosh \frac{L-x}{\ell} & \text { for } \xi \leq x \leq L\end{cases}
$$

is symmetric with respect to $x$ and $\xi$ and satisfies the normalizing condition [30].

The influence of boundaries is illustrated in Figure 7, which shows the weight functions corresponding to a one-dimensional problem solved on the interval $(0, L)$ where $L=20 \ell$. The weight functions $\alpha(x, \xi)$ derived by rescaling (Figure 7a) and $G(x, \xi)$ obtained as the Green function of the Helmholtz-type boundary value problem (Figure $7 \mathrm{~b}$ ) are plotted as functions of $\xi / \ell$ for several fixed values of $x / \ell=0,1,5,10,15,19,20$. For the weight function derived by rescaling [7] of the truncated quartic spline [9], the radius of influence is set to $R=\ell \sqrt{14}$. 
(a)

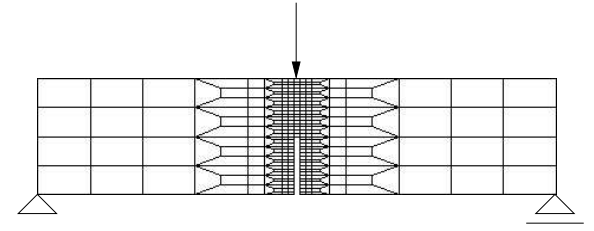

(b)

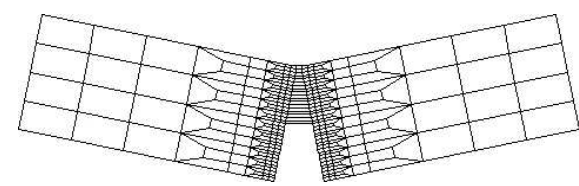

Figure 8. Notched beam under three-point bending: (a) coarsest fi nite element mesh with supports and load, $(b)$ deformed mesh at complete failure (displacements exaggerated)

\section{Examples of failure simulations}

\subsection{Mesh-sensitive results with local damage model}

To demonstrate that a local damage model with softening leads to numerical results that suffer by pathological sensitivity to the discretization, we simulate the three-point bending test of a concrete beam with and without a notch. The beam has a square cross section $100 \times 100 \mathrm{~mm}$ and span $450 \mathrm{~mm}$, and the notch is $5 \mathrm{~mm}$ thick and extends over one half of the beam depth. These dimensions correspond to the experiments performed by Kormeling and Reinhardt (Kormeling et al., 1983).

Failure of the notched beam is first simulated using the finite element mesh in Figure $8 \mathrm{a}$, with minimum element size $5 \mathrm{~mm}$. The elements are standard bilinear quadrilaterals with $2 \times 2$ integration points, and the elastic constants are set to $E=$ $20 \mathrm{GPa}$ and $\nu=0.2$. The adopted damage law [12] corresponds to linear elasticity up to peak stress, followed by exponential softening. If the parameters of the damage law are set to $\varepsilon_{0}=120 \times 10^{-6}$ and $\varepsilon_{\mathrm{f}}=7 \times 10^{-3}$, the simulated peak load is within the experimental bounds and the load-displacement curve favorably compares with experimental data; see Figure 9a. The deformed mesh at the end of the simulation (with exaggerated displacements) is plotted in Figure 8b. It is clear that strain localizes into one vertical layer of elements starting at the notch.

If the material parameters $\varepsilon_{0}$ and $\varepsilon_{\mathrm{f}}$ are kept fixed but the mesh is refined, the results change dramatically. Figure $9 \mathrm{~b}$ shows the numerical load-displacement curves obtained on meshes with minimum element sizes respectively $5 \mathrm{~mm}, 1.67 \mathrm{~mm}$ and $0.556 \mathrm{~mm}$. These element sizes are used in a narrow zone around the axis of symmetry, where the strains are expected to localize, while the other parts of the specimen are discretized by larger elements, to keep the number of unknowns and equations limited. The load-displacement diagram clearly shows that both the peak load and the total dissipated energy (area under the curve) decrease as the mesh is refined. Such a spurious dependence of the results on the discretization is unacceptable. 
(a)

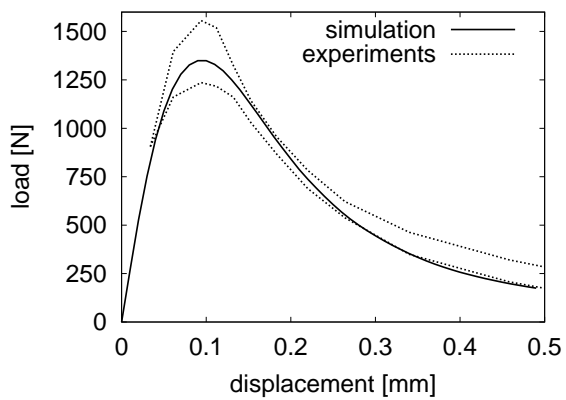

(b)

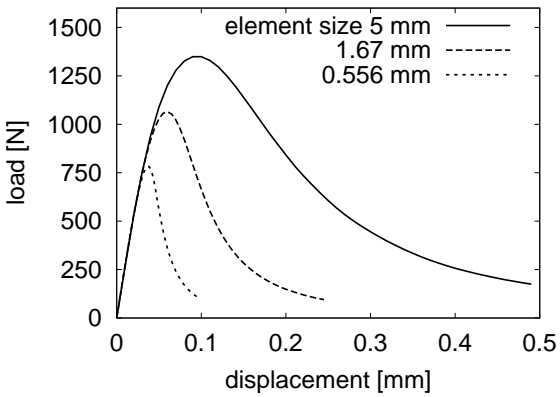

Figure 9. Load-displacement curves for notched beam: (a) simulation on coarse mesh matching experiments, (b) simulations on three different meshes

(a)

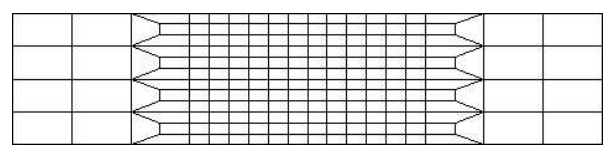

(b)

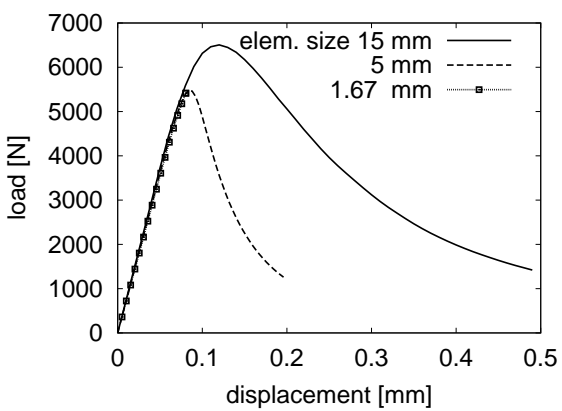

Figure 10. Simulations of unnotched beam: (a) coarsest mesh, (b) load-displacement curves obtained on three different meshes

The same material parameters have been used to simulate the three-point bending test of an unnotched beam with the same dimensions. In this case, damage is initially distributed over a wide region at the bottom face, and so the minimum element size is used in the central third of the beam; see Figure 10a. The test has been run on three meshes with minimum element sizes $15 \mathrm{~mm}, 5 \mathrm{~mm}$ and $1.67 \mathrm{~mm}$, respectively. The load-displacement curves corresponding to the coarse and medium meshes differ substantially, as shown in Figure 10b. Again, mesh refinement from $15 \mathrm{~mm}$ to $5 \mathrm{~mm}$ leads to peak load reduction and to a more brittle response. Upon further refinement to $1.67 \mathrm{~mm}$, the peak load does not decrease any more, but the equilibrium iteration fails to converge at peak. The loss of convergence is due to the abrupt change of the 
(a)

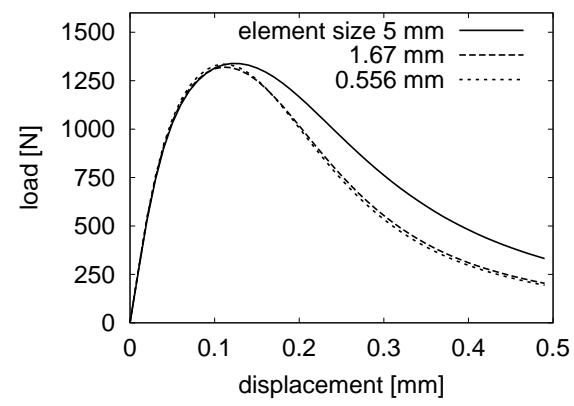

(b)

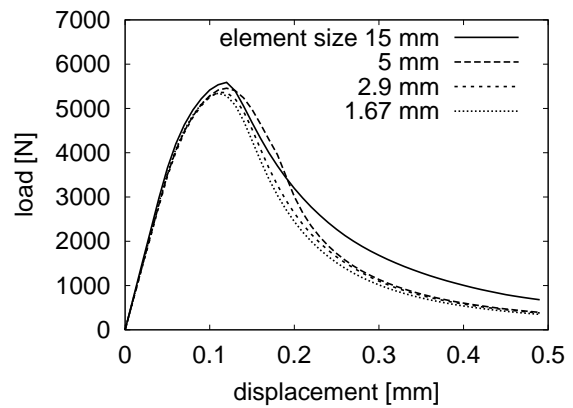

Figure 11. Load-displacement diagrams obtained with the nonlocal damage model: (a) notched beam, (b) unnotched beam

strain increment distribution from a smoothly distributed to a highly localized one. As the mesh gets finer, the number of possible combinations of loading and unloading at individual Gauss points increases and the numerical algorithm has difficulties in finding the actual one.

\subsection{Objective results with nonlocal damage model}

Analysis of the notched beam from Section 5.1 on three different meshes has been rerun using the nonlocal formulation of the isotropic damage model based on nonlocal equivalent strain according to formula [10]. The damage law has again the exponential form [12] with parameters $\varepsilon_{0}=90 \times 10^{-6}$ and $\varepsilon_{\mathrm{f}}=7 \times 10^{-3}$. The nonlocal interaction radius that appears in the definition of the quartic nonlocal weight function [9] has been set to $R=4 \mathrm{~mm}$. The resulting load-displacement curves are plotted in Figure 11a. The curves corresponding to the medium and fine meshes are almost coincident, which indicates that the solution converges upon mesh refinement and confirms that the nonlocal model does not suffer by pathological sensitivity to the mesh size. The load-displacement curve obtained with the coarse mesh is in the post-peak range somewhat above the converged solution. This is normal, because the element size $(5 \mathrm{~mm})$ is larger than the interaction radius $(4 \mathrm{~mm})$ and the localized process zone cannot be resolved with sufficient accuracy.

Analysis of the unnotched beam has been rerun using parameters $\varepsilon_{0}=90 \times 10^{-6}$, $\varepsilon_{\mathrm{f}}=5 \times 10^{-3}$ and $R=8 \mathrm{~mm}$. Note that in this case the element sizes are $15 \mathrm{~mm}$, $5 \mathrm{~mm}, 2.9 \mathrm{~mm}$ and $1.67 \mathrm{~mm}$, and so for $R=4 \mathrm{~mm}$ the medium meshes would still be too coarse and convergence upon mesh refinement could not be convincingly demonstrated. Of course, parameter $R$ should be considered as a material property reflecting the internal length scale of the microstructure (e.g., the size and spacing 
(a)
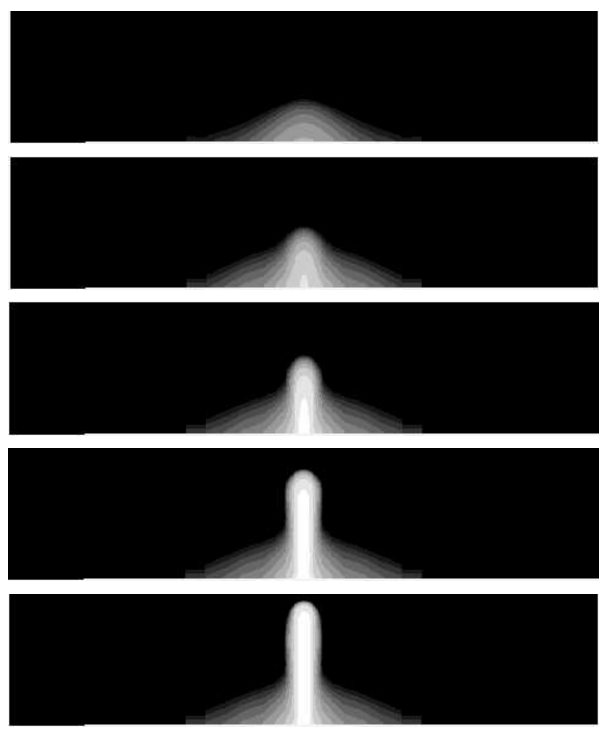

(b)
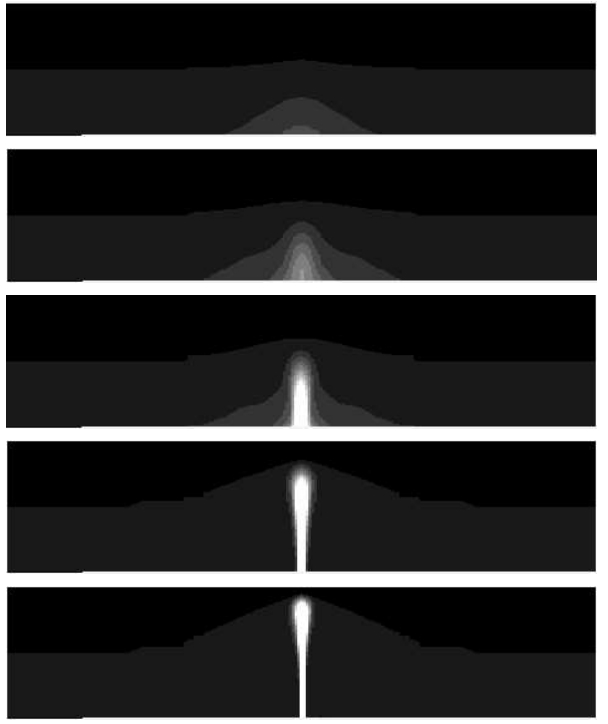

Figure 12. Evolution of the process zone in nonlocal damage simulation of unnotched beam: (a) damage, (b) strain $\varepsilon_{x x}$

of major heterogeneities such as largest aggregates in concrete), but in this academic example we do not try to link it to the actual material. Our aim is to show that the results are almost mesh-independent if the mesh is sufficiently fine. Indeed, the loaddisplacement curves plotted in Figure 11b indicate that two finest meshes give very similar results. The element sizes in the two coarsest meshes are too big compared to parameter $R$ and the nonlocal interaction is not captured accurately. This is why the corresponding load-displacement curves differ from the other two, but the difference is not very dramatic.

The evolution of the process zone simulated on the fine mesh is shown in Figure 12 in terms of damage and strain distribution at five stages of loading. Light shades of gray indicate high levels of the damage parameter $\omega$ and normal strain $\varepsilon_{x x}$ (parallel to the beam axis). Black color marks regions with no damage in the left part of the figure and regions with compressive strain $\left(\varepsilon_{x x}<0\right)$ in the right part. The images in the second row correspond to a state shortly before the peak load and those in the third row to a state shortly after the peak load. Damage is irreversible and it cannot decrease, but in the post-peak range it remains constant in the predamaged region around the bottom face of the beam, except for a narrow band around the axis of symmetry that propagates to the top. The damage band keeps a certain minimum thickness while the 
band of increasing strains becomes progressively thinner. This is quite natural, given that the state at complete failure should represent a macroscopic stress-free crack.

\section{Computational aspects of nonlocal models}

\subsection{Efficient implementation of nonlocal averaging}

Numerical implementation of the nonlocal damage model based on averaging of equivalent strain is relatively straightforward. The evaluation of stresses from given strains remains fully explicit, and no internal iteration loop is needed. Of course, equilibrium iteration on the structural level cannot be avoided, same as for any other nonlinear model. An existing computer code with a certain local damage model can be extended to a nonlocal formulation without excessive effort. All that one needs is to implement the algorithm of weighted spatial averaging and, before damage is evaluated, replace the local equivalent strain by its nonlocal counterpart.

The values of nonlocal equivalent strain must be traced at individual Gauss integration points of the finite element model, because these are the points at which stresses need to be evaluated. Let us denote the coordinates of Gauss points as $\boldsymbol{x}_{k}$, $k=1,2, \ldots N_{G P}$, where $N_{G P}$ is the total number of Gauss points in the finite element model. In the numerical algorithm, the averaging integral [10] is replaced by

$$
\bar{\varepsilon}_{\mathrm{eq}, k}=\sum_{l=1}^{N_{G P}} w_{l} J_{l} \alpha_{k l} \varepsilon_{\mathrm{eq}, l}
$$

where $w_{l}$ is the integration weight of Gauss point number $l, J_{l}$ is the Jacobian of the isoparametric transformation evaluated at this point, and $\alpha_{k l}$ is the weight of nonlocal interaction between points $k$ and $l$, determined as

$$
\alpha_{k l}=\frac{\alpha_{0}\left(\left\|\boldsymbol{x}_{k}-\boldsymbol{x}_{l}\right\|\right)}{\sum_{m=1}^{N_{G P}} w_{m} J_{m} \alpha_{0}\left(\left\|\boldsymbol{x}_{k}-\boldsymbol{x}_{m}\right\|\right)}
$$

It is important to note that $\alpha_{k l}$ vanishes if the distance $\left\|\boldsymbol{x}_{k}-\boldsymbol{x}_{l}\right\|$ between points $k$ and $l$ is larger than the nonlocal interaction radius $R$. So the sums in [37] and in the denominator of [38] do not need to be taken over all Gauss points $l$ but only over those that are located inside the sphere or circle of radius $R$ centered at point $k$. Moreover, since the factors $a_{k l} \equiv w_{l} J_{l} \alpha_{k l}$ are needed at every iteration of every incremental step and do not vary during the simulation, they should be evaluated only once and then stored, rather than recomputed each time they are needed.

An efficient numerical scheme for nonlocal averaging has the following structure:

Loop over all Gauss points $\boldsymbol{x}_{k}, k=1,2, \ldots N_{G P}$.

1) Find all Gauss points $\boldsymbol{x}_{l}$ whose distance from $\boldsymbol{x}_{k}$ is smaller than $R$, and for each of them evaluate $a_{k l}=w_{l} J_{l} \alpha_{0}\left(\left\|\boldsymbol{x}_{k}-\boldsymbol{x}_{l}\right\|\right)$. 
2) Compute the sum $a_{k}=\sum_{l} a_{k l}$.

3) Divide each $a_{k l}$ by $a_{k}$ and store it in a table along with a reference to point $l$.

The table is associated with point $k$.

This procedure is activated as a part of the initialization tasks performed before the actual incremental-iterative solution of the nonlinear problem starts. Each Gauss point is associated with a nonlocal interaction table that gives access to its "neighbors" (i.e., to Gauss points at distance smaller than $R$ ) and to the corresponding interaction coefficients $a_{k l}$.

The search for nonlocal neighbors can be done by checking the distance between all pairs of Gauss points, but for larger problems this step can become a bottleneck of the computation. A much more efficient implementation is based on the quadtree (in two dimensions) or octree (in three dimensions) technique. The entire body is placed in a rectangle or cube that is hierarchically divided into smaller rectangles or cubes down to the required level, and this structure is stored in a tree. Each Gauss point is assigned to one of the leafs of the tree, depending on its position in space. The search for neighbors can then be restricted to a limited number of leafs that are easily identified and accessed.

The stress evaluation procedure repeatedly called during the incremental-iterative solution makes use of the nonlocal interaction tables when the nonlocal equivalent strain (or any other nonlocal variable used by the specific nonlocal model) is computed. To obtain $\bar{\varepsilon}_{\text {eq, } k}$, it is sufficient to get the local equivalent strains $\varepsilon_{\text {eq }, l}$ at all points $l$ that are included in the table associated with point $k$, multiply each of them by the coefficient $a_{k l}$ and take a sum of these products.

Nonlocal averaging requires a certain extra effort as compared to the corresponding local model, but the convergence of the equilibrium iteration on the global level is usually more regular for the nonlocal model than for the local one. The reason is that the spatial distribution of strain is relatively smooth even after the onset of localization, and the transition from a diffuse damage pattern to a localized one is not so abrupt. Consequently, the increased numerical effort per iteration is partially compensated by the reduced number of iterations needed to restore equilibrium. Since the nonlocal model completely removes the pathological sensitivity to the mesh size and also at least partially alleviates the mesh-induced directional bias, this extra effort is indeed worthwhile.

\subsection{Simulations on adaptive meshes}

Nonlocal models lead to smooth solutions with a continuous distribution of strain. However, to resolve narrow bands of highly localized strains, it is necessary to use sufficiently fine computational grids. Fortunately, the mesh needs to be fine only in the damage process zone, while the remaining part of the structure can be reasonably represented by a coarser discretization. The localization pattern is in general not known in advance, and it is extremely tedious to construct suitably refined meshes "by 

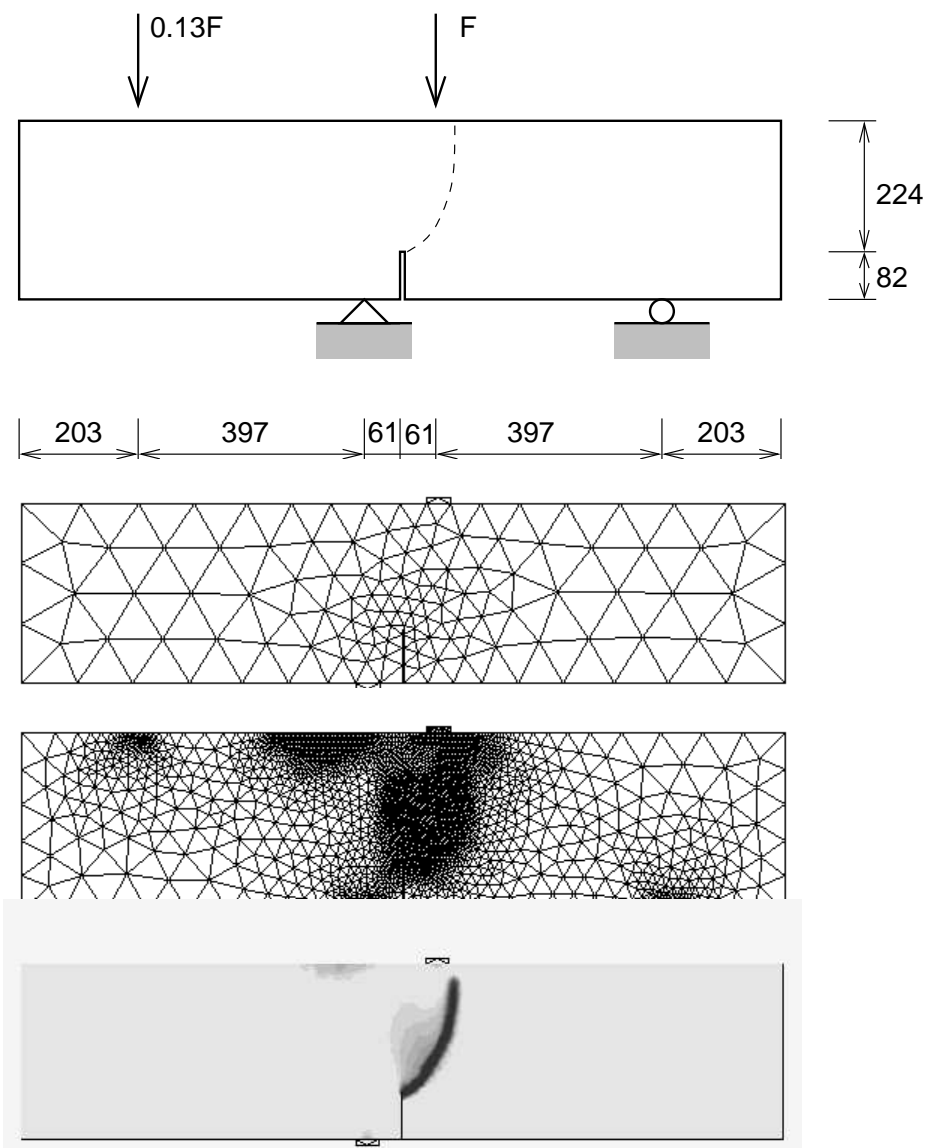

Figure 13. Adaptive analysis of a four-point shear test using a nonlocal anisotropic damage model: specimen geometry and loading, initial mesh, fi nal mesh, and fi nal damage zone

hand". Efficiency of the analysis can be greatly increased by an adaptive technique that automates the whole process.

Details of the mesh-adaptive strategy applied to nonlocal damage models are discussed in (Patzák et al., 2004). As an example, we present the results of an adaptive simulation of the four-point shear specimen in Figure 13a tested by Arrea and Ingraffea (Arrea et al., 1982). The test is simulated using the nonlocal version of a certain anisotropic damage model denoted as MDM, which combines the microplane theory with the principle of energy equivalence; see (Jirásek, 1999). During the adaptive simulation, the initial mesh with 145 nodes and 234 elements (Figure 13b) is gradually transformed into the final mesh with 4174 nodes and 8120 elements (Figure 13c). The damage distribution at complete failure, depicted in Figure 13d, nicely corresponds to 

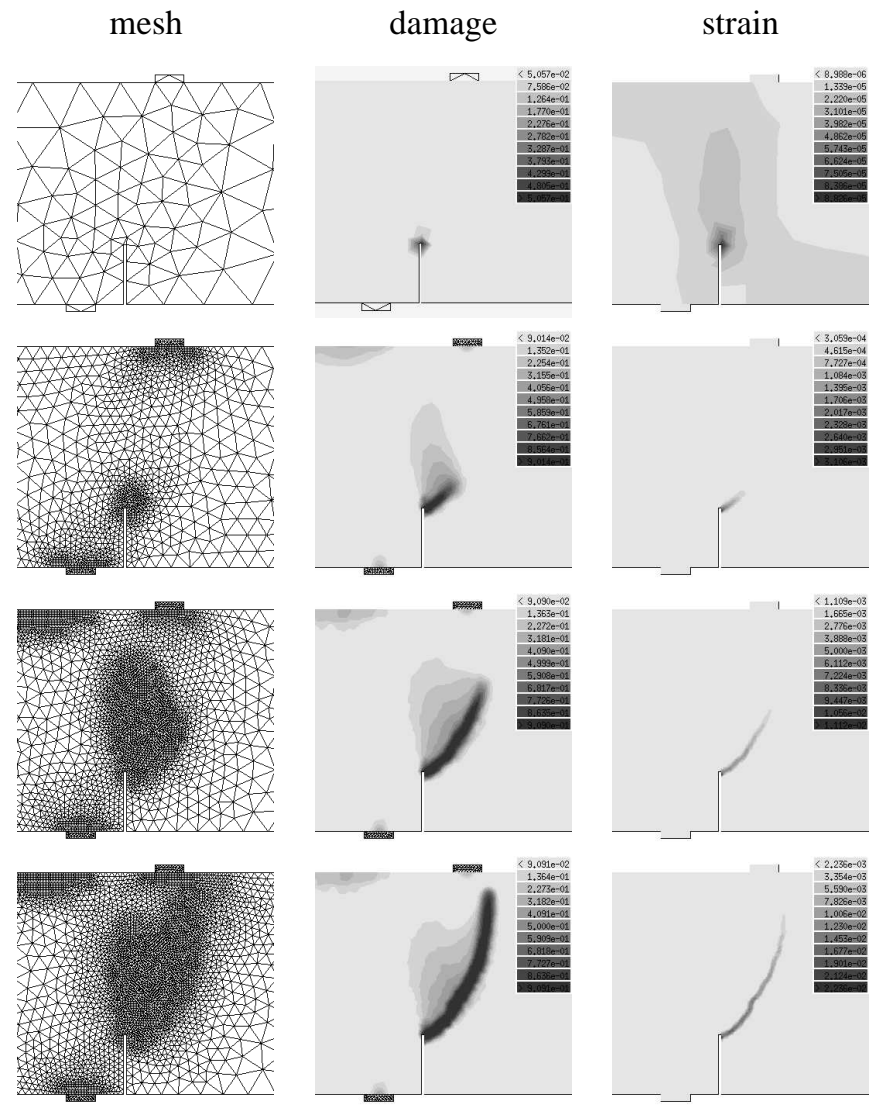

Figure 14. Adaptive analysis of a four-point shear test using the nonlocal anisotropic damage model: evolution of the mesh, damage indicator and maximum principal strain in the central part of the beam

the experimental results. The evolution of the mesh and of the damaged zone is shown in Figure 14.

\section{Acknowledgements}

Financial support of the Ministry of Education of the Czech Republic under Research Plan MSM 6840770003 is gratefully acknowledged. 


\section{References}

Aifantis E. C., "On the microstructural origin of certain inelastic models", Journal of Engineering Materials and Technology, ASME, vol. 106, 1984, p. 326-330.

Arrea M., Ingraffea A. R., Mixed-mode crack propagation in mortar and concrete, Department of Structural Engineering n ${ }^{\circ}$ 81-83, Cornell University, Ithaca, NY, 1982.

Bažant Z. P., Jirásek M., "Nonlocal integral formulations of plasticity and damage: Survey of progress", Journal of Engineering Mechanics, ASCE, vol. 128, 2002, p. 1119-1149.

Bažant Z. P., Lin F.-B., "Nonlocal smeared cracking model for concrete fracture”, Journal of Engineering Mechanics, ASCE, vol. 114, 1988a, p. 2493-2510.

Bažant Z. P., Lin F.-B., “Nonlocal yield-limit degradation”, International Journal for Numerical Methods in Engineering, vol. 26, 1988b, p. 1805-1823.

Bažant Z. P., Luzio G. D., "Nonlocal microplane model with strain-softening yield limits", International Journal of Solids and Structures, vol. 41, 2004, p. 7209-7240.

Bažant Z. P., Ožbolt J., "Nonlocal microplane model for fracture, damage, and size effect in structures", Journal of Engineering Mechanics, ASCE, vol. 116, 1990, p. 2485-2505.

Borino G., Failla B., Parrinello F., "A Symmetric Formulation for Nonlocal Damage Models", in H. A. Mang, F. G. Rammerstorfer, J. Eberhardsteiner (eds), Proceedings of the Fifth World Congress on Computational Mechanics (WCCM V), Vienna University of Technology, Vienna, Austria, 2002. ISBN 3-9501554-0-6, http://wccm.tuwien.ac.at.

Eringen A. C., "On nonlocal plasticity”, International Journal of Engineering Science, vol. 19, 1981, p. 1461-1474.

Eringen A. C., "Theories of nonlocal plasticity”, International Journal of Engineering Science, vol. 21, 1983, p. 741-751.

Jirásek M., "Nonlocal models for damage and fracture: Comparison of approaches", International Journal of Solids and Structures, vol. 35, 1998, p. 4133-4145.

Jirásek M., "Comments on microplane theory", in G. Pijaudier-Cabot, Z. Bittnar, B. Gérard (eds), Mechanics of Quasi-Brittle Materials and Structures, Hermès Science Publications, Paris, 1999, p. 55-77.

Jirásek M., "Mathematical analysis of strain localization", Revue Européenne de Génie Civil, vol. 11, 2007, p. 977-991.

Jirásek M., Rolshoven S., "Comparison of integral-type nonlocal plasticity models for strainsoftening materials", International Journal of Engineering Science, vol. 41, 2003, p. 15531602.

Jirásek M., Zimmermann T., Rotating crack model with transition to scalar damage: I. Local formulation, II. Nonlocal formulation and adaptivity, LSC Internal Report 97/01, Swiss Federal Institute of Technology, Lausanne, Switzerland, 1997.

Kormeling H. A., Reinhardt H. W., Determination of the fracture energy of normal concrete and epoxy modifi ed concrete, Technical Report n ${ }^{\circ} 5-83-18$, Stevin Lab, Delft University of Technology, 1983.

Mazars J., Application de la mécanique de l'endommagement au comportement non linéaire et à la rupture du béton de structure, Thèse de Doctorat d'Etat, Université Paris VI., France, 1984. 
Ožbolt J., Bažant Z. P., "Numerical smeared fracture analysis: Nonlocal microcrack interaction approach”, International Journal for Numerical Methods in Engineering, vol. 39, 1996, p. 635-661.

Patzák B., Jirásek M., “Process zone resolution by extended fi nite elements”, Engineering Fracture Mechanics, vol. 70, 2003, p. 957-977.

Patzák B., Jirásek M., “Adaptive resolution of localized damage in quasibrittle materials”, Journal of Engineering Mechanics, ASCE.

Peerlings R. H. J., de Borst R., Brekelmans W. A. M., de Vree J. H. P., "Gradient-enhanced damage for quasi-brittle materials", International Journal for Numerical Methods in Engineering, vol. 39, 1996, p. 3391-3403.

Pijaudier-Cabot G., Bažant Z. P., "Nonlocal damage theory”, Journal of Engineering Mechanics, ASCE, vol. 113, 1987, p. 1512-1533.

Planas J., Guinea G. V., Elices M., Basic issues on nonlocal models: uniaxial modeling, Technical Report $n^{\circ}$ 96-jp03, Departamento de Ciencia de Materiales, ETS de Ingenieros de Caminos, Universidad Politécnica de Madrid, Ciudad Universitaria sn., 28040 Madrid, Spain, 1996.

Polizzotto C., "Remarks on some aspects of nonlocal theories in solid mechanics", Proceedings of the 6th National Congress SIMAI, Chia Laguna, Italy, May, 2002. CD-ROM.

Simone A., "Explicit and implicit gradient-enhanced damage models", Revue Européenne de Génie Civil, vol. 11, 2007, p. ??

Strömberg L., Ristinmaa M., "FE-formulation of a nonlocal plasticity theory", Computer Methods in Applied Mechanics and Engineering, vol. 136, 1996, p. 127-144.

Vermeer P. A., Brinkgreve R. B. J., "A new effective non-local strain measure for softening plasticity", in R. Chambon, J. Desrues, I. Vardoulakis (eds), Localisation and Bifurcation Theory for Soils and Rocks, Balkema, Rotterdam, 1994, p. 89-100. 


\section{ANNEXE POUR LE SERVICE FABRICATION \\ A FOURNIR PAR LES AUTEURS AVEC UN EXEMPLAIRE PAPIER \\ DE LEUR ARTICLE ET LE COPYRIGHT SIGNE PAR COURRIER \\ LE FICHIER PDF CORRESPONDANT SERA ENVOYE PAR E-MAIL}

1. ARTICLE POUR LA REVUE :

REGC - 11/2007. Damage and fracture in geomaterials

2. Auteurs :

Milan Jirásek

3. TITRE DE L'ARTICLE :

Nonlocal damage mechanics

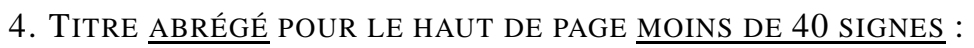

Nonlocal damage mechanics

5. DATE DE CETTE VERSiON :

September 17, 2007

6. CoOrdonnéEs des Auteurs :

- adresse postale :

Department of Mechanics

Faculty of Civil Engineering

Czech Technical University in Prague

16629 Prague, Czech Republic

Milan.Jirasek@epfl.ch

- téléphone : +420-224354481

- télécopie : +420-224310775

- e-mail : milan.jirasek@fsv.cvut.cz

7. LOGICIEL UTILISÉ POUR LA PRÉPARATION DE CET ARTICLE :

IATEX, avec le fichier de style article-hermes2.cls, version $1.23 \mathrm{du}$ 02/08/2006.

8. FORMULAIRE DE COPYRIGHT :

Retourner le formulaire de copyright signé par les auteurs, téléchargé sur : http://www. revuesonline.com

SERVICE ÉDITORIAL - HERMES-LAVOISIER 14 rue de Provigny, F-94236 Cachan cedex

Tél. : 01-47-40-67-67

E-mail : revues@lavoisier.fr

Serveur web : http://www.revuesonline.com 